\title{
TEXTURE AND ANISOTROPY OF PLASTIC PROPERTIES IN DEEP DRAWING STEELS
}

\author{
D. DANIEL and J. J. JONAS \\ Department of Metallurgical Engineering, McGill University \\ 3450 University Street, Montreal, Canada, H3A 2A7.
}

The textures of different types of deep drawing steels were measured and analyzed using the series expansion method. The angular variation of $r$ value in the rolling plane was calculated from the ODF coefficients by means of different models. Comparison with experimental data shows that, in the case of sharp textures, an intermediate relaxed constraint model (pancake version) is a better predictor of the plastic strain ratio than the extreme Taylor or Sachs-Kochendorfer type calculation. This is justified as being more favorable energetically for the texture components present in deep drawing steels. The best quantitative agreement is obtained when the CRSS ratio for glide on the $\{112\}<111>$ and $\{110\}<111>$ slip systems is 0.95 (except for the AKDQ and IF2 grades, for which values of 0.90 and 1.0, respectively, are preferred).

\section{INTRODUCTION}

The prediction of $\mathbf{r}$-values using the series expansion method is generally carried out within the framework of the Taylor model. ${ }^{1}$ In this paper, methods based on the Sachs-Kochendörfer and intermediate "relaxed constraint" grain interaction models are developed and employed in order to improve the accuracy of predictions, as has been done for the simulation of deformation textures. 2 Measurements were made on thirty-one commercial drawing quality low carbon steels and compared with the predictions, so that the most appropriate hypotheses for the description of bcc polycrystal deformation can be selected. Yield stress anisotropies and yield surface sections were also calculated. It is shown how the different hypotheses affect yield surface shape and can thus influence formability predictions such as forming limit diagrams. ${ }^{3}$

\section{EXPERIMENTAL WORK}

Thirty-one commercial low-carbon steel sheets of five different types were studied: (1) batch annealed Al-killed drawing quality (AKDQ) steel, (2) 
commercial grade rimmed steel, (3) high strength low alloy (HSLA) steel, and (4) and (5) two types (differing in their drawability) of interstitial-free extra low carbon steel. The textures were determined by means of X-ray measurements, using standard reflection techniques. The ODF analysis provided $\mathrm{C}_{1}{ }^{\mu \nu}$ coefficients for even $l$ up to 22 . Ghost correction, carried out by the iterative positivity method, 4 was employed to provide the complete ODF, i.e. to calculate the $C_{1}{ }^{\mu \nu}$ coefficients for odd $l$.

Tensile tests were carried out on specimens cut from the sheets at several orientations with respect to the rolling direction, i. e. every $15^{\circ}, 22.5^{\circ}$ or $45^{\circ}$. In order to achieve high accuracy $(< \pm 0.1)$ in $r$-value, the measurements were made on tapered samples. 5,6

\section{MODELING r-VALUE MEASUREMENT}

Plasticity calculations on bec materials involve questions regarding the slip systems that are operative and the critical resolved shear stresses (CRSS's) associated with each of these systems. Both experimental and theoretical studies support the view that the crystallographic description of glide on $\{110\}$ and $\{112\}$ planes in the $<111>$ directions is sufficient for the description of plastic flow. Another feature of bcc deformation is the existence of asymmetric slip on the $\{112\}$ planes. In that description, the CRSS ratios $\alpha_{S}=\tau\{112\}$ twinning $/ \tau\{110\}$ and $\alpha_{H}=\tau\{112\}$ anti-twinning $/ \tau_{\{110\}}$ characterize single crystal plastic deformation. Experimental values reported in the literature include $\alpha_{\mathrm{S}} \approx 1.0$ and $\alpha_{\mathrm{H}} \approx 1.04$ to 1.14 for decarburized Fe single crystals. 7 From experiments on Fe-3wt.\% Si single crystals, Orlans-Joliet et al. obtained values of $\alpha_{\mathrm{S}} \approx 0.93$ and $\alpha_{\mathrm{H}} \approx 0.96 .8$ Due to the uncertainties regarding these values in the case of low carbon steels, several hypothetical sets of $\alpha_{\mathrm{s}}$ and $\alpha_{\mathrm{H}}$ were used in this study.

The following grain interaction models were systematically tested: the Taylor (FC), Sachs-Kochendörfer (SK) and relaxed constraints (RC) models. Four RC models were evaluated: lath $\left(\dot{\varepsilon}_{13}\right.$ relaxed), RC ${ }_{4}\left(\dot{\varepsilon}_{23}\right.$ relaxed), $R C_{3}\left(\dot{\varepsilon}_{13}\right.$ and $\dot{\varepsilon}_{23}$ relaxed, i.e. pancake) and $\mathbf{R C}\left(\dot{\varepsilon}_{12}, \dot{\varepsilon}_{13}\right.$ and $\dot{\varepsilon}_{23}$ relaxed). The application of the series expansion method to the simulation of $r$-value measurement is described in References 1,5 and 6 .

In addition to the existing grain interaction assumptions, a new model,6,9 referred to as $\mathrm{LW}$ for least work, was also tested. It involves the combination of the $\mathrm{FC}$ and $\mathrm{RC}_{3}$ deformation modes in proportions which depend on the grain orientation g. Analysis of the single crystal plastic work $M_{r}(g)$ functions for different CRSS ratios and various $r$-values shows that the average relative difference $\Delta$ between $M_{r}(g)_{R C 3}$ and $M_{r}(g)_{F C}$ (i. e. $\left.\Delta=\left(M_{r}(g)_{R C 3}-M_{r}(g)_{F C}\right) / M_{r}(g)_{F C}\right)$ is about $11 \%$ and that $1 / 3$ of the grain orientations in Euler space have $\Delta$ values of less than $6 \%$. The above two values were chosen to define a least work criterion in which $1 / 3$ of the grain orientations deform according to the $\mathrm{FC}$ model, $50 \%$ by the $\mathrm{RC}_{3}$ deformation mode and the remaining ones select the $\mathrm{FC}$ or $\mathrm{RC}_{3}$ mode with a probability depending on the $\Delta$-value. 


\section{MODELING RESULTS}

\section{Influence of Alternative Descriptions of bec Plastic Deformations}

Texture data from the thirty-one low carbon steels were used to predict $r$-values for different CRSS ratios and the FC, lath, $\mathrm{RC}_{4}, \mathrm{RC}_{3}, \mathrm{RC}_{2}$ and SK grain interaction models. The predictions, using several $\alpha_{S}$ and $\alpha_{H}$ values, for two representative IF2 and AKDQ examples, are compared with the experimental results in Figure 1. The calculated value of $r$ decreases from the case of restricted $\{110\}<111>$ glide (curve (1)) to that of restricted $\{112\}<111>$ slip (curve (0)). It was also observed that when a difference between twinning and antitwinning was allowed for $\left(\alpha_{S} \neq \alpha_{H}\right)$, the predictions corresponded to those with equal CRSS's in the two senses, with $\alpha$ approximately equal to the average of $\alpha_{\mathrm{S}}$ and $\alpha_{\mathrm{H}}$, especially for realistic $\alpha$ values less than 1 . It is apparent that a $5 \%$ change of this ratio around the value $\alpha=1$ affects the calculated $\vec{r}$-values for the AKDQ and IF steels by 10 to $15 \%$, except for the $\mathrm{RC}_{4}$ model (about $5 \%$ ). It was also observed that the $\overline{\mathbf{r}}$ values predicted for the weak textures associated with the RIM and HSLA steels are much less sensitive to a similar change in CRSS ratio (about 5\%).

The predictions should first be assessed on the basis of the agreement between the shapes of the experimental and theoretical $r(\theta)$ curves. A conclusion regarding the best CRSS ratio for good quantitative agreement is more difficult to draw because of the error associated with the predicted $\mathbf{r}$ values (minimization of the Taylor factor, i.e. of the average $\bar{M}_{r}$ functions). However, because of the number of steels studied, clear tendencies are observed. Before comparing the predictions from the various models, it appears that an $\alpha$-value of 1.0 is most suitable for $r$-value prediction in the IF2 steels. Also, a value of 0.95 is preferred for the IF1 grades and restricted slip on the $\{112\}$ planes or an $\alpha$-value of 0.9 appears to give relatively good agreement with experimental results in the AKDQ steels. These trends confirm the experimental evidence according to which the presence of interstitial elements in solution makes slip more difficult on the $\{110\}$ planes. 7,10

Comparison Between the r-Value Predictions and the Experimental Results

The r-value predictions for a typical steel selected from the groups studied are presented in Figure 2. The calculations were carried out using the six models in turn with the appropriate CRSS ratio, as discussed in the above section. As expected from the results for the common ideal orientations (see references 5 and 6 ), the choice of model is particularly important for the IF and AKDQ steels. These steels are the ones employed for deep drawing applications, and for which the $r$-value is therefore of interest. In the case of the 'weak' textures, i.e. those of the RIM and HSLA steels, the models differ by the $\overline{\mathbf{r}}$ predicted and not by the shape of the $\mathbf{r}(\theta)$ curve. This is related to the observation that the predictions according to the different models are similar for orientations such as the Goss (rimmed steel) 
and the $\{100\}<011>$ (HSLA steel). Furthermore, as mentioned in the above section, a change of CRSS ratio in the range 0.95 to 1.05 does not significantly affect the calculated $r$-values for these two cases. So in order to decide on the most suitable model, it is best to examine the cases of the IF and AKDQ steels.

Analysis of the set of steels studied shows that the $\mathrm{RC}_{3}$ model gives the best overall agreement with the experimental measurements, although selected models can lead to particularly good fits for certain steels in a given range (i.e. the $\mathrm{RC}_{4}$ model for the $\mathrm{AKDQ}$ steels and $\theta$ less than $45^{\circ}$, the lath model for the HSLA and RIM steels, and $\mathrm{RC}_{2}$ for the IF2 ones).

No significant differences (within the \pm 0.1 range of uncertainty in $r$ value) were observed between the $\mathrm{RC}_{3}$ and $\mathrm{LW}$ calculations for a given CRSS ratio. This reflects the fact that, for most of the texture components encountered in deep drawing steels, the $\mathrm{RC}_{3}$ deformation mode satisfies the least work criterion defined above. Maps of the preferred deformation modes for orientations belonging to the $\phi_{2}=45^{\circ}$ section of Euler space are presented in Figure 3. It can be seen that for $r$ ranging from 0 to 2.5 (i. e. $q$ from 0 to 0.7 ), only the orientations near $\{111\}<112>$ (such as $\{554\}<225>$ and $\{223\}<472>$ ), among the main texture components near the $\gamma$-fibre, deform according to the FC mode or to a mixture of $\mathrm{FC}$ and $\mathrm{RC}_{3}$. This result provides an argument in favour of the use of the $\mathrm{RC}_{3}$ model. The $\mathrm{LW}$ criterion, which supports this deformation mode, seems especially satisfactory when the instantaneous plastic deformation of approximately equiaxed polycrystals is concerned (i.e. the 'pancake' model seems to apply even when the grains are equiaxed).

For the vicinity of the $\gamma$-fibre in low carbon steels, the RC deformation mode appears as a good compromise between the "extremely" relaxed $\mathrm{SK}$ and $\mathrm{RC}_{2}$ ones, which call for complex accommodations at the grain boundaries, and the FC method, which is energetically unfavourable. The lath and $\mathrm{RC}_{4}$ calculations are justified when the elongated microstructures induced by high strain rolling deformation are of concern.

\section{Yield Surface Predictions}

As observed above for the $\mathrm{r}(\theta)$ results, the $\mathrm{RC}_{3}$ model is the most appropriate for representation of the yield stress anisotropy in the rolling plane.5,6 The differences between the $r$-value and yield stress variations predicted by the various models reflect subtle differences in the associated yield loci. A two dimensional section, determined (by the method described in Reference 11) from the FC, $\mathrm{RC}_{3}$ and $\mathrm{LW}$ models, is displayed in Figure 4.

The shape differences can be examined in terms of the ratio $B$ of the plane strain flow stress $\left(\dot{\varepsilon}_{22}=0\right), \sigma_{w}$, to the plane strain flow stress $\left(\dot{\varepsilon}_{33}=0\right), \sigma_{f}$ Some results of this type are presented in Figure 5 for one IF2 and one AKDQ steel. This stress ratio was shown to be empirically related to the limiting drawing ratio $(L D R)(\ln (L D R)=n \beta) .12$ The latter relation was established for radially isotropic textures, i. e. $\beta=$ constant. However, the mechanical analysis can be extended to the present case by simply assuming 
that failure in drawing occurs along the direction where $\beta$ is a minimum. Considering an efficiency coefficient $\eta$ of 0.7512 and $\beta_{\min }$ as the critical value, the predicted LDR for the IF2 grade is about 2.3 in the FC calculation and 2.4 in the $\mathrm{RC}_{3}$ one. No experimental data were available for the present steels. However, it appears that the LDR for extra deep drawing steels (IF2 type) is significantly higher than the ones measured in ordinary mild steels (in the range 2.1-2.3). Thus the $\mathrm{RC}_{3}$ predictions again seem more realistic than the $\mathrm{FC}, \mathrm{RC}_{4}$ or lath ones.

\section{CONCLUSIONS}

A decrease in the CRSS ratio (which increases the proportion of $\{112\}$ slip with respect to $\{110\}$ glide) lowers the predicted $r$-values. The calculations show that a ratio of 1.0 is suitable for IF2 steels, while values of 0.95 and 0.9 are preferred for, respectively, the IF1 and AKDQ grades; this tendency is in agreement with observations reported in the literature.

Comparison between the experimental data and the predictions of $r$ value reveals that the Taylor and Sachs-Kochendörfer models are unsuitable for these purposes, especially for the sharp textures present in IF and $\mathrm{AKDQ}$ steels. Conversely, the $\mathrm{RC}_{3}$ calculations show good agreement; this can be justified by 'least work' considerations.

\section{REFERENCES}

1. H. J. Bunge, M. Schulze and D. Grzesik : Peine / Salzgitter Berichte, (Sonderheft,1980).

2. P. Van Houtte, Proc. of the $7_{\text {th }}$ Int. Conf. on Textures of Materials, (Netherlands Soc. Mater. Sci., Zwijndrecht, 1984), pp. 7-23.

3. D. W. Lin, D. Daniel and J. J. Jonas, submitted to Metall. Trans.

4. H. J. Bunge, Proc. of the 8th Int. Conf. on Textures of Materials, (AIME, Warrendale, 1988), pp. 69-78.

5. D. Daniel and J. J. Jonas, Metall. Trans., 1990, 21 A, pp. 331-343.

6. D. Daniel, Ph. D. Thesis, McGill University, Montreal, Canada, 1990.

7. P. Franciosi, Acta Metall., 1983, 31, pp. 1331-42.

8. B. Orlans-Joliet, J. H. Driver and F. Montheillet, Acta Metall., 1990, 38, pp. 581-594.

9. L. Toth, J. J. Jonas, D. Daniel and R. K. Ray, Metall. Trans., 1990, in press.

10. T. Senuma, H. Yada and R. Shimizu, Submitted to Acta Metall.

11. P. Van Houtte, Textures and Microstructures, 1987, 7, pp. 29-72.

12. W. F. Hosford, Formability: Analysis, Modeling and Experimentation, (AIME, New York, 1978), p.78. 

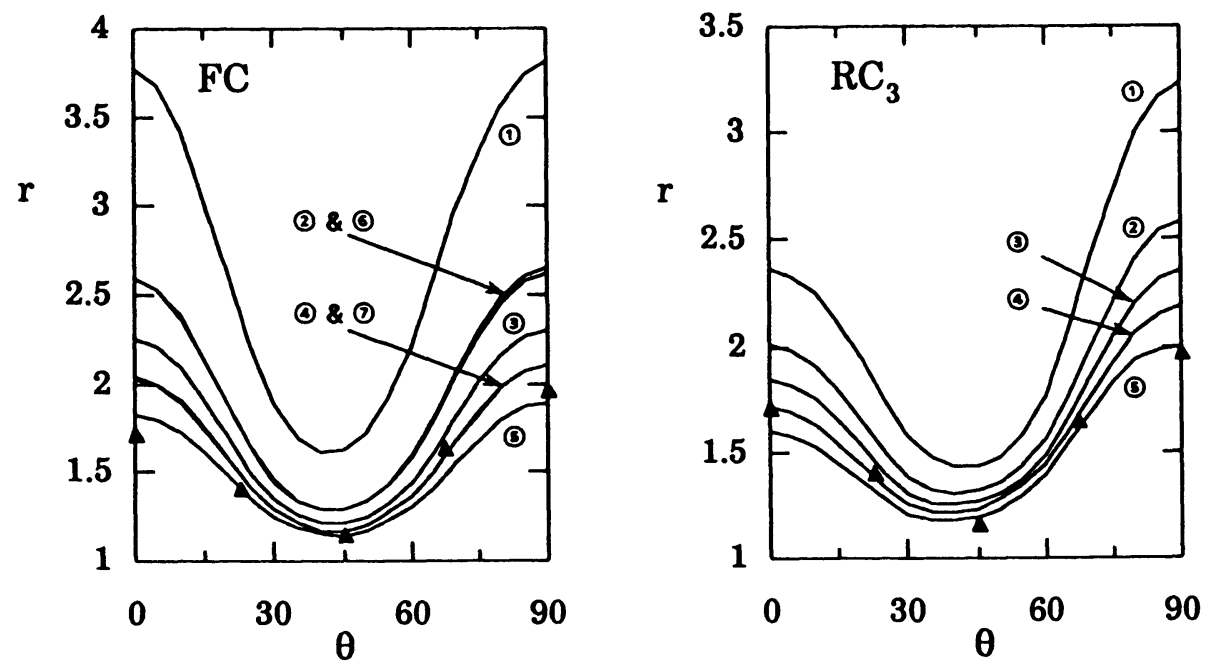

Figure 1 : Influence of CRSS ratio on the values of $r(\theta)$ obtained from the FC and $\mathrm{RC}_{3}$ models for an AKDQ steel. Experimental $r$-values are indicated by ( $(\Delta)$. (1): $\alpha_{\mathrm{S}}, \alpha_{\mathrm{H}}>1.15$; (2): $\alpha_{\mathrm{S}}=\alpha_{\mathrm{H}}=1.05$; (3): $\alpha_{\mathrm{S}}=\alpha_{\mathrm{H}}=1.0$; (4) $\alpha_{S}=\alpha_{H}=0.95$; (5): $\alpha_{S}, \alpha_{H}<0.87 ;$ (6) $\alpha_{S}=1.0, \alpha_{H}=1.1 ;$ ( ) $\alpha_{S}=0.93, \alpha_{H}=0.96$.
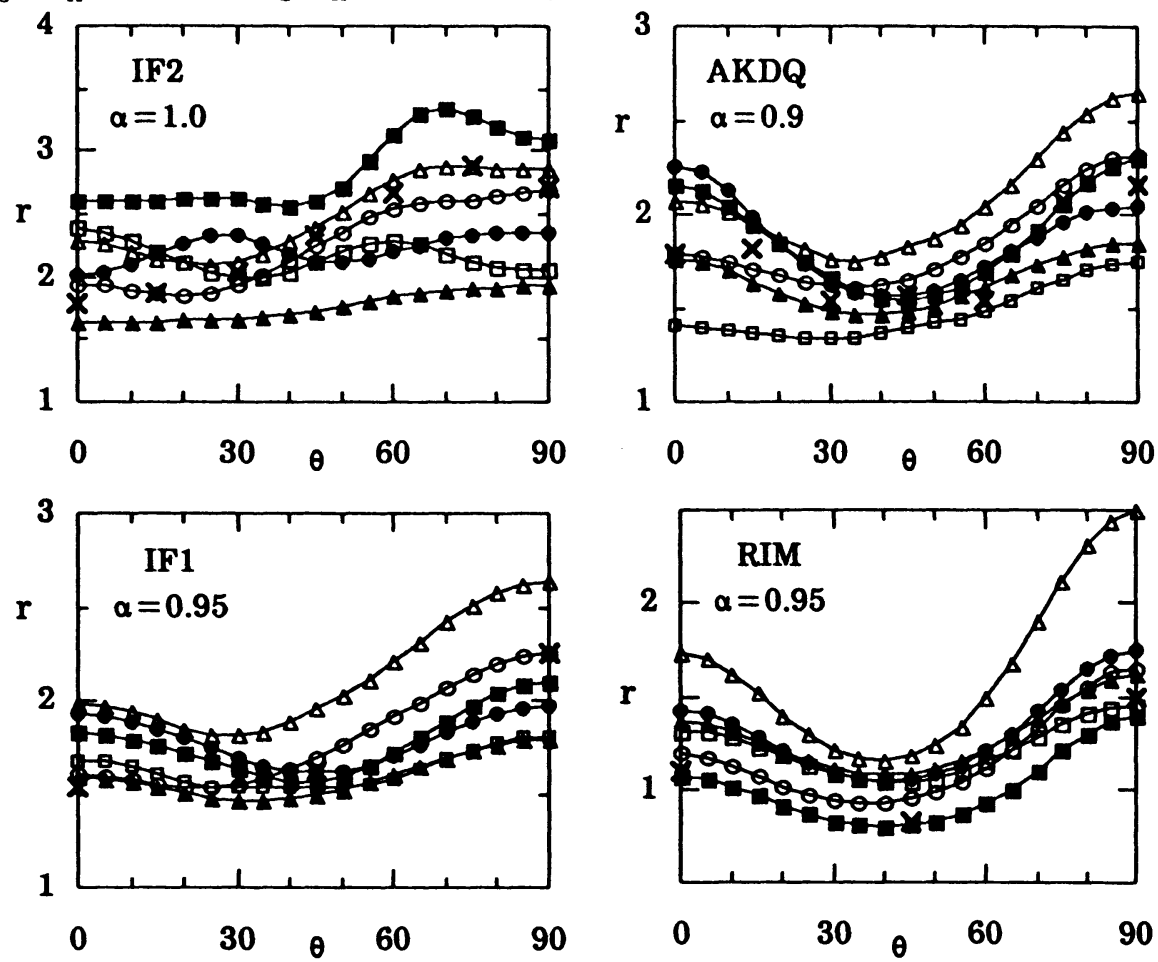

Figure 2 : Comparison between experimental data $(x)$ and the $r(\theta)$ predictions obtained from the $\mathrm{FC}(\bullet)$, lath $(\square), \mathrm{RC}_{4}(\Delta), \mathrm{RC}_{3}(0), \mathrm{RC}_{2}(\Delta)$ and SK (ㅁ) models. 

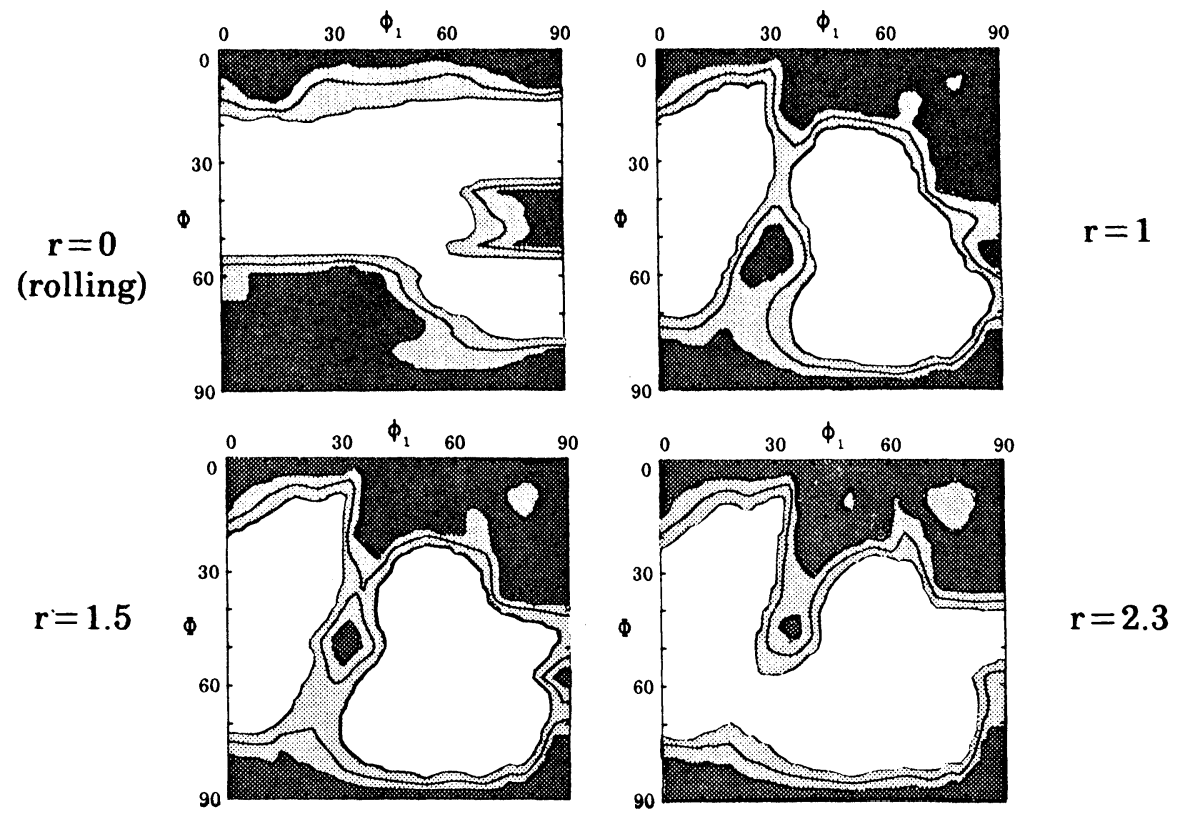

Figure 3 : Maps illustrating the preferred deformation modes called for by the LW model: $\mathrm{RC}_{3}(\square), \mathrm{FC}(\square)$ or mixed FC-RC $(\square)$. The grain orientations being considered are located in the $\Phi_{2}=45^{\circ}$ section and have been subjected to various strain paths characterized by their $r$-values. The line within the mixed $\mathrm{FC}-\mathrm{RC}_{3}$ areas indicates the $50 \% \mathrm{FC}-50 \% \mathrm{RC}_{3}$ limit.

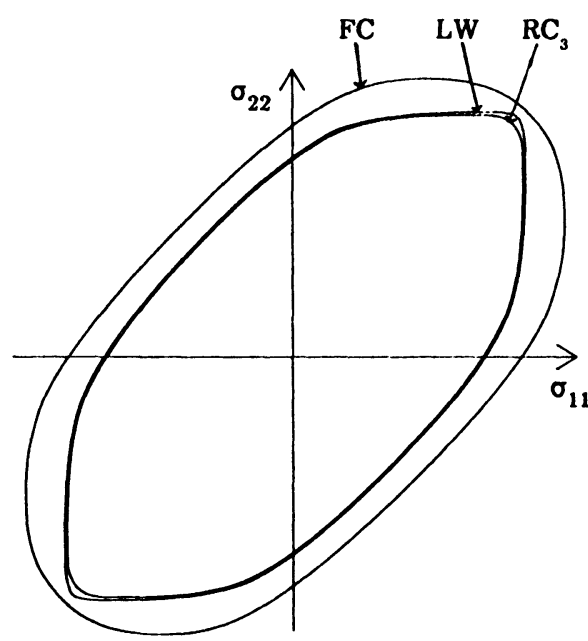

Figure 4: $\sigma_{11}-\sigma_{22}$ yield surface sections calculated for an AKDQ steel by the $\mathrm{FC}, \mathrm{RC}_{3}$ and $\mathrm{LW}$ models. The $x_{1}$ direction coincides with RD.

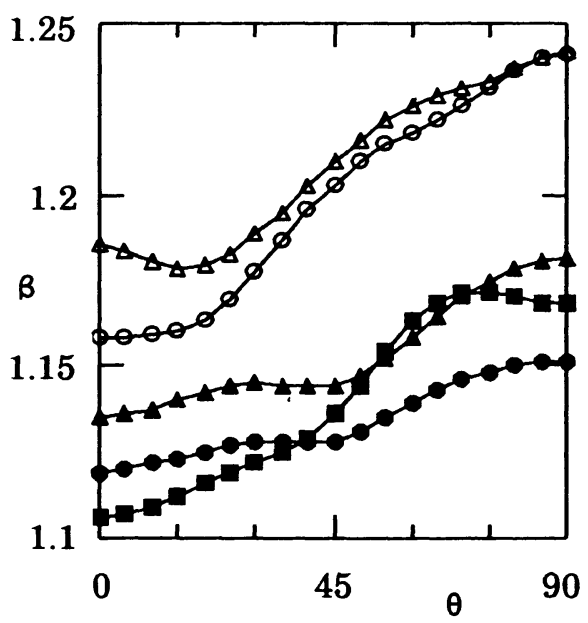

Figure 5 : Comparison between the $B(\theta)$ predictions obtained from the

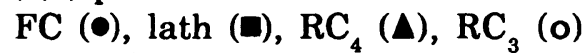
and $\mathrm{RC}_{2}(\Delta)$ models for an IF2 grade. 